\title{
A European Centre for Infectious Diseases
}

During the past year or so a lively discussion has ensued in the correspondence columns of several scientific journals on the subject of a European Centre for Infectious Diseases (ECID). The need for such a centre was first proposed by Michel Tibayrenc (1997 Nature 389: 433-434, Parasitology Today 13: 454). The proposal was further refined at a meeting of the international board of scientific advisers for the project which was held at the IRD (The French agency for scientific research in developing countries) centre in Montpellier on the 11th and 12th September, 1998 (J Bradbury 1998, Lancet 352: 969, D Butler 1998 Nature 393:106).

The proposed centre is inspired by the United States CDC (Centres for Disease Control and Prevention) and in particular the National Centre for Infectious Diseases (NCID) and would have a triple mission: (i) advanced research (ii) surveillance, control and prevention (iii) teaching/training. However opposition to this idea has also been expressed (Editorial 1998, Lancet 352: 1237, J Giesecke \& J Weinberg, Lancet 352: 1308). Everyone agrees that etiological agents of infectious disease show no respect for international frontiers and are thus a natural subject for international cooperation. Opponents of the ECID proposal think that the combat of emerging and re-emerging infectious diseases can be achieved by a network of existing national centres and electronic communication. The ECID proposal however while it envisages a "centre with walls" also includes the need for outstations and collaborative centres in the different countries so that in fact the two positions are not so divergent.

An important aspect of the ECID proposal that has only been briefly mentioned in the above discussions is the establishment of privileged links with developing countries. The international advisory board for the project contains several members from infectious disease centres in these countries and in the discussions in Montpellier the following points were emphasised:

Collaboration with the developing countries should not be stereotyped. There are great differences (economic, cultural, social and academic) not only among these countries but also among the regions of many countries, among institutions within the same region and even among departments within the same institution. Therefore there is unlikely to be one standard model for collaboration however there may be some general principles that can be applied.

Collaboration must be for mutual benefit. Types of collaboration to be avoided are "aid" type programs where all plans and priorities are set by the donor organization without consultation with the recipient and where there is just a unidirectional flow of funds and ideas. Another type of collaboration to avoid is a "colonial" type program where the developing country institute merely becomes a source of material whether clinical samples (sera, urine or biopsies), pathogenic microorganisms or vector samples. A real collaboration would involve bi-directional flow of ideas and techniques as well as scientific advance and human resources development for both partners.

Collaboration is unlikely to be successful if it is only initiated as a result of a formal agreement at any level (presidential, ministerial or institutional). In collaborating with developing countries it is important to initiate projects between individuals who are already known to each other and have already established a personal relationship and a basis for trust and technical cooperation. Formal agreements are important but should be a product and not a starting point of collaboration.

The training/research to be provided by ECID should be holistic in character. The institute should have a critical size that would allow the multidisciplinary study of infectious disease, including all the parameters of the transmission chain. The "centre with walls" would foster daily interaction between bench scientists, epidemiologists, experts in fieldwork, clinicians and public health workers. The facilities would offer access to powerful new technologies such as DNA chips however traditional expertise in disciplines such as microbiology, parasitology and entomology would also be stressed. The training would stress pragmatic over academic goals.

Among the international collaborations that can be envisaged are: emergency responses, to deal with outbreaks of known and unknown diseases, involving the ability of ECID to respond quickly with equipment and supplies as well as multidisciplinary human resources (such as entomologists, microbiologists, epidemiologists, infectious disease physicians and public health workers); long term collaboration with selected institutions in developing countries in building infrastructure to combat emerging and reemerging infectious diseases in endemic areas.

In these respects the ECID would be a welcome addition to organizations such as CDC and WHO in the control of infectious diseases. A steering committee for the ECID project has been appointed and the project will be discussed at further meetings in 1999. Further details about the project can be found at the website http://cepm.mpl.orstom.fr/ 